\title{
Editorial
}

Nephrology

Published online: September 3, 2018

\section{A New Treatment for Calciphylaxis in Hemodialysis Patients?}

\author{
Carmine Zoccali ${ }^{\mathrm{a}}$ Francesca Mallamaci ${ }^{\mathrm{a}, \mathrm{b}}$ \\ ${ }^{a}$ CNR-IFC, Clinical Epidemiology and Pathophysiology of Hypertension and Renal Diseases, Reggio Calabria, Italy; \\ ${ }^{b}$ Nephrology, Hypertension and Renal Transplantation Unit, Reggio Calabria, Italy
}

The randomized trial is at the top of the hierarchy of study designs for testing the effects of treatments. Prospective and retrospective studies and case-control studies come after the randomized trial and case-series and case reports are at the bottom of the list. However, as for discovery the ranking is just the opposite, with case-series and case reports at the top and the randomized trial at the bottom [1]. Indeed, new ideas are often generated by observations made in a single patient or in small series of patients or by perusing sparse information in clinical databases. The astute clinician and the knowledgeable scientist recognize what is unique or new in these observations and the new idea flashes in his/her mind. New ideas are the sparks of discovery. Study designs of increasing complexity are then applied to test for causality, from case-control and retrospective and prospective studies to the randomized clinical trial which is the definitive, undisputed test for proving causal effects.

Calcific uremic arteriolopathy or calciphylaxis is a rare, systemic disease which mainly occurs in patients on chronic dialysis. The main feature of this disease is a relentless calcification of arterioles, thrombosis, and ischemia. Risk factors for this disease include hyperparathyroidism, inappropriate vitamin $\mathrm{D}$ use, diabetes, inflam-

\section{KARGER}

(๑) 2018 S. Karger AG, Basel

E-Mail karger@karger.com

www.karger.com/ajn mation, low levels of inhibitors of calcification, and warfarin. In a survey based on 1,030 American hemodialysis patients with calciphylaxis, as much as 144 patients (14\%) were being treated with warfarin at the time of diagnosis and the risk of the disease conveyed by warfarin (OR 3.22) was substantially higher than that by hyperparathyroidism, the use of vitamin $\mathrm{D}$ compounds, and diabetes [2]. Warfarin is the cornerstone for the prevention of thrombotic events in atrial fibrillation. Given the high prevalence of this alteration in the dialysis population (7-27\%) [3] the exposure to this drug is a quite common risk factor for calciphylaxis. Vascular calcification by warfarin is due to its inhibitory effect of on vitamin K-dependent matrix Gla protein synthesis, a potent calcification inhibitor (Fig. 1). On the other hand, warfarin itself might trigger microvascular thrombosis because it decreases protein $S$ secretion in endothelial cells by more than $90 \%$ thereby generating a pro-coagulant environment at endothelial cell level [4]. Such an effect is in contrast with the powerful systemic anti-coagulant effect of the same drug and may actually contribute to arteriolar thrombosis. However, this hypothetic effect still remains to be proven in human studies. On the other hand, arteriolar damage by microvascular calcification - that is, the 
Fig. 1. Interference of warfarin and novel oral anticoagulant drugs on mechanisms underlying calcific uremic arteriolopathy. The figure is commented in the main text.

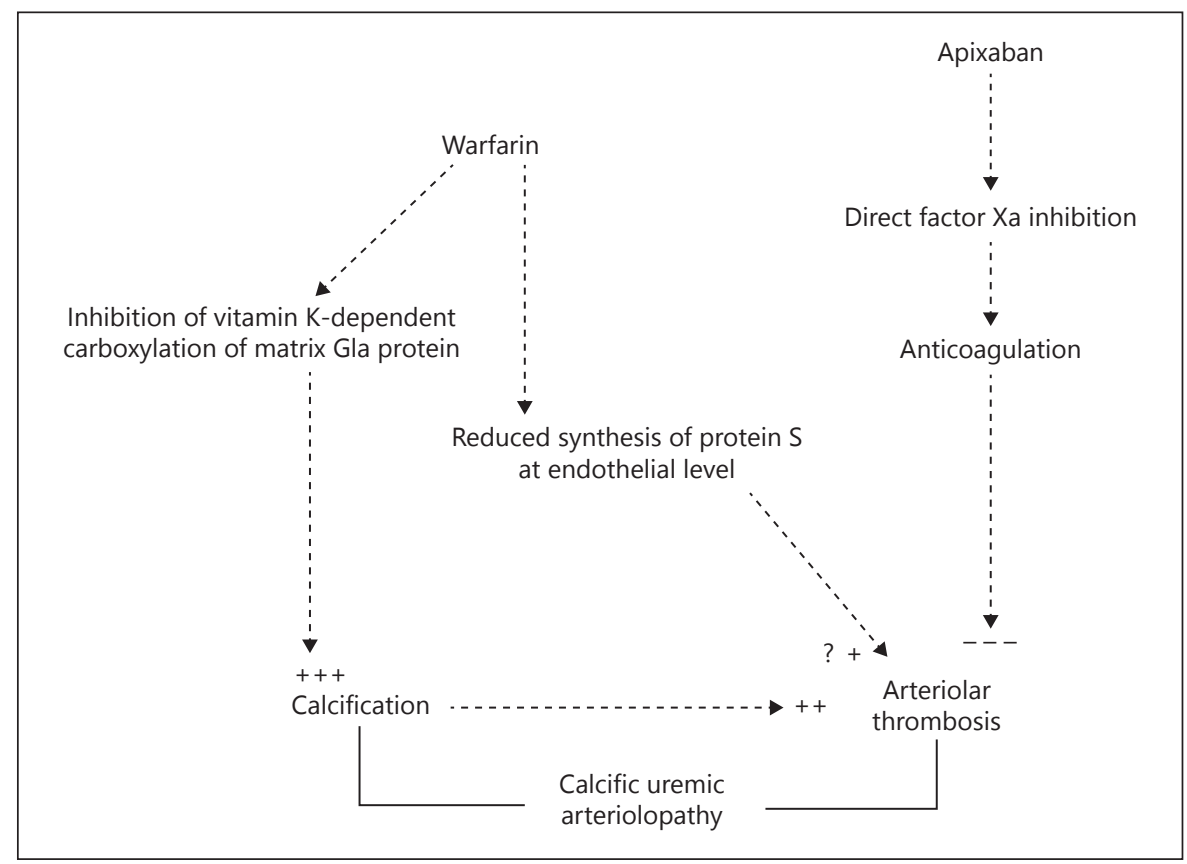

main alteration triggered by warfarin - is per se a procoagulant mechanism. For the foregoing, it is formally recommended that the use of warfarin be discontinued in patients with calciphylaxis [5]. In theory, novel oral anticoagulant (NOA) drugs appear a suitable alternative to warfarin for anticoagulation in hemodialysis patients with calciphylaxis. However, the effectiveness and the safety of these drugs in these patients is unknown.

Such a complete lack of knowledge on a question of major clinical relevance, led Shah et al. [6] to mining information on the use of NOA in calciphylaxis from a large clinical data base. As alluded to, observations in case series may generate discoveries and stimulate studies applying more rigorous research designs. The emerging hypothesis is that a NOA blocking factor Xa, apixaban, may prevent thrombosis (Fig. 1) and retard the evolution of arteriolopathy in hemodialysis patients with this devastating disease. The vast majority of patients in Shah's study had been treated with warfarin before receiving apixaban and this drug not only prevented new thrombotic episodes but also improved the lesions of calciphylaxis while warfarin notoriously worsens these lesions. The obvious limitations of this case-series are the short follow-up (about 6 months) and the lack of a parallel series of patients maintained on standard anti-calciphylaxis treatment. Their low rank in the ladder of evidence notwithstanding [1], matched case-series represent a more robust approach than case-series to explore the efficacy of treatments in rare diseases. The authors repeatedly em- phasize that calciphylaxis has a dim prognosis with a $50 \%$ yearly mortality rate. However, this figure reflects the average mortality risk by calciphylaxis in a very large, national (USA) dialysis network collecting data in a multitude of dialysis centers while the case-series described in the present issue of the Journal [6] reflects experience in 2 hospitals affiliated with a prestigious academic center, the Harvard University, whose renal units are headed by investigators with a lasting clinical and scientific interest on calciphylaxis. Case-series collected at academic centers applying multimodal treatments showed a high survival rate and registered relevant improvements in the signs and symptoms of this disease [7]. In Shah et al., study apixaban use was associated with an improvement in the manifestations of the disease in the $90 \%$ of cases and with a survival ( $80 \%$ at 1-year ) almost identical to the average survival rate in the USRDS. Given the very short life expectancy of calciphylaxis, this is a truly remarkable finding. In another recent study in 16 patients with calciphylaxis treated with apixaban or other NOA about onethird of patients had complete resolution of cutaneous lesions [8].

The clinical management of rare diseases is far less investigated than that of common diseases and for this reason the scientific evidence underlying the treatment of these conditions is almost always insufficient. If true, the clinical benefits of apixaban, particularly the improvements of cutaneous lesions, are of magnitude that can be captured in a randomized clinical trial of relatively small 
dimensions. Needless to say that such a trial should carefully standardize background treatments for calciphylaxis and randomize patients to 2 groups, one treated by apixaban and the other by a matched placebo. Testing NOA in calciphylaxis would be of obvious clinical relevance because thrombotic arteriolopathy is a key pathomechanism in this rare, deadly disease. Registries of calciphylaxis which are being built both in the USA and in Europe may generate the ideal environment for launching clinical trials embedded in clinical practice in this under-investigated disease.

\section{Disclosure Statement}

The authors declare that there are no conflicts of interest to disclose.

\section{References}

1 Vandenbroucke JP: Observational research, randomised trials, and two views of medical science. PLoS Med 2008;5:e67.

2 Nigwekar SU, Zhao S, Wenger J, Hymes JL, Maddux FW, Thadhani RI, et al: A nationally representative study of calcific uremic srteriolopathy risk factors. J Am Soc Nephrol 2016; 27:3421-3429.

3 Wetmore JB, Mahnken JD, Rigler SK, Ellerbeck EF, et al: The prevalence of and factors associated with chronic atrial fibrillation in Medicare/Medicaid-eligible dialysis patients. Kidney Int 2012;81:469476.
4 Stern D, Brett J, Harris K, Nawroth P: Participation of endothelial cells in the protein Cprotein $S$ anticoagulant pathway: the synthesis and release of protein S. J Cell Biol 1986; 102:1971-1978.

5 Nigwekar SU, Thadhani R: Calciphylaxis (calcific uremic arteriolopathy). 2018. https://www.uptodate.com/contents/calciphylaxis-calcific-uremic-arteriolopathy?sea rch=calciphylaxis adult\&source $=$ search result \&selected Title $=1 \sim 39 \&$ usage type $=$ default $\&$ display_rank $=1$ (cited June 14, 2018).
6 Garza-Mayers AC, Shah R, Sykes DB, Nigwekar SU, Kroshinsky D: The successful use of apixaban in dialysis patients with calciphylaxis who require anticoagulation: a retrospective analysis. Am J Nephrol 2018;48:168-171.

7 Russo D, Capuano A, Cozzolino M, Napolitano P, Mosella F, Russo L, et al: Multimodal treatment of calcific uraemic arteriolopathy (calciphylaxis): a case series. Clin Kidney J 2016;9:108-112.

8 King BJ, El-Azhary RA, McEvoy MT, Shields RC, McBane RD, McCarthy JT, et alDirect oral anticoagulant medications in calciphylaxis. Int J Dermatol 2017;56:1065-1070. 